\title{
Comparing the content of lipids derived from the eye lenses of various species
}

\section{Tomasz Panz, Magdalena Lepiarczyk, Agnieszka Żuber}

\author{
Department of Biophysics, Faculty of Biochemistry, Biophysics and Biotechnology, \\ Jagiellonian University, Krakow, Poland
}

\begin{abstract}
The lipid content in the eye lens was analyzed and compared among various species in this study. The eye lens lipids of the following species were investigated: cow, horse, duck, and freshwater trout. Additionally, the lipids derived from cataractous bovine lens and from cataractous human eye lens lipoprotein complexes were analyzed. The following lipid classes were detected in clear lenses: cholesterol, sphingomyelin, phosphatidylcholine, phosphatidyletanolamine, and phosphatidylserine. In cataractous bovine lens and in lipoprotein complexes from human nuclear cataract, phosphatidyloinositol and phosphatidyloglycerol were detected. Cholesterol and sphingomyelin, essential for hypothetical formation of cholesterol-rich domains, were the most abundant lipids in the lenses of all investigated species. These two components of eye lens lipid fraction were analyzed quantitatively using thin layer chromatography and spectrophotometric assay; the other lipids were identified qualitatively using thin layer chromatography. (Folia Histochemica et Cytobiologica 2011; Vol. 49, No. 3, pp. 425-430)
\end{abstract}

Key words: eye lens, cataract, cholesterol, sphingomyelin, thin layer chromatography

\section{Introduction}

The eye lens is a unique part of both the human and the animal body, different in many aspects from any other soft tissue. The main feature of the normal lens, crucial for sight, is its transparency.

It seems that natural selection guided eye lens features towards a structure and components capable of preserving transparency of the lens for many years, giving awareness of the possible presence of reactive species and exposure to visible and UV radiation [1]. One of the lens components are lipid membranes present in densely packed fiber cells. Studying the lipids in these membranes might provide information that would explain some properties of eye lens membranes of various species. For example, the correlation between saturation of the eye lens lipids and longevity of animals has been noted by investigators [2].

Correspondence address: T. Panz, Department of Biophysics, Faculty of Biochemistry,

Biophysics and Biotechnology, Jagiellonian University, Gronostajowa Str. 7, 30-387 Krakow, Poland;

e-mail: tomasz.panz@uj.edu.pl
One of the most striking features of lens lipids is their high cholesterol content [3]. In most cases, the high cholesterol in eye lens membranes correlates with high sphingomyelin content [4]. Unlike in any other part of the human body, the eye lens lipids in membranes gradually change their content proportions with age, increasing sphingomyelin content, and decreasing phosphatidylcholine and plasmalogens content [5]. When the homeostasis of the lens is impaired, a loss of transparency occurs. Such a pathology is called a cataract [6]. Most investigations concerning the causes of cataracts focus on the changes in lens proteins [7] but there are some data indicating that not only proteins, but also lipids, may play an important role in cataract development [8]. The impairment of cholesterol synthesis and decrease of its content in the eye lens of rats, dogs and humans is connected with cataract onset [9]. This hypothesis is supported by the observations of side effects of drugs inhibiting cholesterol synthesis [8].

The aim of the present study was a closer look at the cholesterol and sphingomyelin content in the eye lens of different species, in order to find out lipid content patterns specific to animals from different environments, and also to determine the changes in lipid 
contents following the development of the cataract. Revealing the changes occurring in lens lipids following the development of a cataract might help in finding possible means of cataract prevention, by modification of lipid metabolism.

\section{Material and methods}

Chemical reagents. Chemical reagents and organic solvents were of analytical grade and were obtained from the following sources:

Salts for buffer preparation, acetic acid, sulfuric acid POCh, Gliwice, Poland

Phosphomolybdic acid spray reagent - SIGMA (catalog number P-4869), cholesterol - SIGMA (catalog number C-8253), DPPC = D1- $\alpha$-phosphaditylcholine dipalmoityl - SIGMA (catalog number P-5911), EYPC = L-A-phosphaditylcholine from egg yolk type XI-E - SIGMA (catalog number P-2772), SM = sphingomyelin from chicken egg yolk — Fluka (catalog number 85615), OPA — phtalodialdehyde - Fluka (catalog number 79760), reagent for cholesterol determination - Alpha Diagnostics, Poland; plates for thin layer chromatography Silica Gel 60 - Merck (catalog number HX 894971)

Biological material. The experiments were performed with eye lenses obtained from various species. The bovine and equine eyes were collected from slaughterhouses in either Tarnow or Slomniki (Poland). The eyes from ducks were obtained from farms in the vicinity of Michalow, Poland. The eyes from the freshwater trout were obtained directly from a fish-farm.

Immediately after the animal's death, the eyes were collected and placed into a styrofoam box filled with ice. The eyes were then transported to the laboratory and dissected. The lenses were separated from other parts of eye, flushed up with phosphate buffered saline, frozen and stored at $-80^{\circ} \mathrm{C}$ until experiments.

The human lipoprotein complexes from cataractous lenses were obtained from the Ophthalmic Clinic in Krakow as post-operative material from operations performed by the cataract nucleus expulsion procedure. The lipoprotein complexes were used as the source of lipids derived by the procedure of lipid extraction [10].

Lipid extraction. Lipid extraction was based on the Folch procedure [10]. In brief, the tissue was homogenized (glass-glass homogenizer) with organic solvent mixture (chloroform-methanol 2:1), keeping the solvent:tissue ratio 20:1 ( $\mathrm{vol} / \mathrm{vol})$ and washed by centrifugation. Washing was repeated a few times with new portions of solvent mixture. The chloroform fractions, enriched with lipids, were collected, pooled and dried in a rotary evaporator. The dry lipids were weighed, dissolved in chloroform and stored in small amber-glass laboratory bottles at $-20^{\circ} \mathrm{C}$.
Thin layer chromatography (TLC). The lipids derived from eye lens membranes were separated by TLC on the same plates as lipid standards. The lipid standards were placed in separate starting points on the plate with a microsyringe, as chloroform solutions. The dry mass of lipid standard added for one point was within the range of 5 to $30 \mu \mathrm{g}$. The lens lipid samples were placed in lines, up to $1 \mathrm{~cm}$ long, and the dry mass of lipids for one line was $50 \mu \mathrm{g}$. Two different solvents mixtures were used:

1. Chloroform:methanol:acetic acid:water (81:10:45:4, vol/ $/ \mathrm{vol} / \mathrm{vol} / \mathrm{vol}$ ) - used to separate the phospholipids from cholesterol and free fatty acids.

2. Chloroform:methanol:7 $\mathrm{M} \mathrm{NH}_{3}{ }^{*} \mathrm{H}_{2} \mathrm{O}$ (45:35:10, vol/ /vol/vol) - used to separate the different classes of phospholipids.

The run was performed until the front of the solvent reached the top of the plate. Then the plate was removed from the TLC chamber and dried by leaving it under the air flow cabinet for about an hour.

Development of lipids on the plate was performed in one of two ways:

1. The dried plate was soaked in the following solvent: 50 $\mathrm{mg}$ of $\mathrm{FeCl}_{3} * 6 \mathrm{H}_{2} \mathrm{O}$ dissolved in $90 \mathrm{ml}$ of water, with $5 \mathrm{ml}$ of acetic acid and $5 \mathrm{ml}$ of $96 \%$ sulfuric acid and then dried in the laboratory dryer in $100^{\circ} \mathrm{C}$ for $2-3$ minutes.

2. The dried plate was soaked in $10 \% \mathrm{w} / \mathrm{v}$ solution of phosphomolybdenic acid in ethanol (SIGMA, catalog number P-4869), then placed into a laboratory dryer and left there at $100^{\circ} \mathrm{C}$ for 10 minutes. After a few hours, the yellowish color from phosphomolybdenic acid was bleached, and dark purple or blue spots of cholesterol and phospholipids appeared on the plate.

Quantitative analysis of cholesterol. 1. Spectrophotometric method of cholesterol determination based on OPA (o-phtaldialdehyde) [11]. Preparation of OPA reagent: $5 \mathrm{mg}$ of OPA was dissolved in $10 \mathrm{ml}$ of glacial acetic acid and vortexed for 10 minutes. Sample preparation: $1 \mu$ of stock lipid solution extracted from the eye lens of a given species was added to Eppendorf tube (stock: $10 \mathrm{mg}$ of lipids in $1 \mathrm{ml}$ of chloroform). The solvent was evaporated under the nitrogen flow. $1 \mathrm{ml}$ of OPA solution and $0.5 \mathrm{ml}$ of concentrated sulfuric acid was added to the tube. Then the tube was vortexed and left for 15 minutes at room temperature. The absorbance of each sample was measured at $552 \mathrm{~nm}$ against the blank. The results were compared to a standard curve, prepared with known amounts of cholesterol.

2. Enzymatic method of cholesterol determination. For this test, the ready-to-use fluid reagent (Alpha-Diagnostic, Poland) suitable for automated tests for cholesterol determination in blood plasma was used $[12,13]$. Routinely the reagent is suitable for cholesterol determination in blood. For our purposes, to make in vitro measurements of eye lens lipids possible and reliable, the lipids had to be prepared as multilamellar liposomes. The end product of en- 
zyme reactions involving cholesterol is red chinoine, measured at $500 \mathrm{~nm}$. The red-pink product of the reaction is stable for about 30 minutes.

Multilamellar liposome preparation: at first, the lipids had to be prepared in the form of dry film on the walls of the glass test tubes. To achieve this, the organic solvent was removed with nitrogen flow, followed by drying the samples under vacuum for at least one hour. The $0.5 \mathrm{ml}$ of phosphate buffered saline was added to each test tube with lipid film and vortexed for $30 \mathrm{~min}$ in elevated temperature (up to $50^{\circ} \mathrm{C}$ ). Additionally, five cycles of freezing in liquid nitrogen and thawing in warm water-bath of the liposome suspension were performed, to ensure complete removal of lipids from the test tube walls.

Sample preparation: $5 \mu \mathrm{l}$ of liposome suspension was added to the dry test tube and $500 \mu$ l of cholesterol reagent was added, the content was shaken vigorously and incubated for $5 \mathrm{~min}$ at $37^{\circ} \mathrm{C}$. Then the sample was measured spectrophotometrically at $500 \mathrm{~nm}$ against the blank.

Quantitative determination of sphingomyelin in lens lipids. 1. Indirect determination of sphingomyelin by total phosphorus measurements (based on the Fiske-Subbarow method) [14]. In the sphingomyelin (SM) molecule there is one atom of phosphorus. If the sample contains only SM as the source of phosphorus, the content of SM molecules might be calculated from the contents of phosphorus.

Sample preparation: the lipids derived from various species were separated on the plates using the TLC method. The sphingomyelin spots were scraped off the surface of the plate (together with silica gel) using a stainless steel spatula. The silica gel with sphingomyelin was placed into glass test tubes, and $0.5 \mathrm{ml}$ of $5 \mathrm{M} \mathrm{H}_{2} \mathrm{SO}_{4}$ was added to each tube. All samples were placed into a hot oven $\left(180^{\circ} \mathrm{C}\right)$ for 1.5 hours. If the samples were black after that treatment, they were taken out of the oven, cooled down and the additional $200 \mu$ l of concentrated $\mathrm{HNO}_{3}$ was added. The burning was then continued for an additional $30 \mathrm{~min}$ utes, until the color changed to yellowish. After cooling, $2 \mathrm{ml}$ of water was added to each sample and all samples were placed into a boiling water-bath for 10 minutes to convert pyrophosphate to orthophosphate. After cooling the samples, they were centrifuged for $5 \mathrm{~min}$ at $540 \mathrm{~g}$. From each sample, $1.5 \mathrm{ml}$ of supernatant was taken, without disturbing the bottom silica gel pellet. The $1.5 \mathrm{ml}$ portion of supernatant was transferred into calibrated $10 \mathrm{ml}$ glass test tubes and to each of them $4.5 \mathrm{ml}$ of water, $0.8 \mathrm{ml}$ of ammonium molybdate and $0.4 \mathrm{ml}$ of eikonogen reagent were added. All tubes with samples were filled with water up to $10 \mathrm{ml}$. They were mixed and placed for 10 minutes into $37^{\circ} \mathrm{C}$ water bath. Then all samples were measured at 658 $\mathrm{nm}$ against the blank.

2. Densitometric determination of sphingomyelin content in lens lipid samples. Known quantities of sphingomy- elin in stock solution ranging from $5 \mu \mathrm{g}$ up to $50 \mu \mathrm{g}$ (4-5 points) were placed at the starting line of TLC plate. Actual sample - lipids from eye lens, about $70 \mu \mathrm{g}$, was placed next to calibration curve spots. The thin layer chromatography run was performed, and the lipid spots were developed. Then the scan of the whole TLC plate with white light was performed using a FLUOR-S densitometer (Bio-Rad). The intensity of each spot of sphingomyelin was measured using Quantity One software. The quantity of sphingomyelin was calculated from the slope of the calibration curve and recorded intensity of the spot coming from the samples of lens lipids.

\section{Results}

The thin layer chromatography Rf coefficients measured for lipids extracted from the lenses of various species are presented in Table 1.

The $R_{f}$ coefficients recorded for eye lens lipids from different species in most cases fall into a range of values, indicating that particular lipids in TLC run do not appear like small points, but rather large spots. The $\mathrm{R}_{\mathrm{f}}$ coefficients for the same lipid species (columns in Table 1) are virtually the same, which confirms the reproducibility of the TLC method applied for lipids derived from different sources. We saw the absence of two lipids in cataractous bovine lens and in material derived from human cataractous lens, namely phosphatidylethanolamine and phosphatidylserine in opposition to the lipid content pattern detected for other, non-cataractous material. Two lipid species, namely phosphatidyloinositol and phosphatidyloglycerol, were not detected in material from transparent lenses and were present in samples derived from the cataractous material.

The cholesterol determination in eye lens lipids from various species is shown in Tables 2 and 3.

The content of cholesterol derived from lenses derived by each method for separate experiments indicates the variation of this parameter. The highest variation was detected for samples derived from the cataractous material.

The averaged results of cholesterol assays are shown on Figure 1.

The cholesterol content measured with two methods shows general agreement of results, although the values obtained by enzymatic assay are always smaller than those obtained by the OPA method. For mammals, the cholesterol content falls within the range of 20-30\%; for ducks it is roughly one third of that size (about 8.5\%); and for freshwater trout it is about $4 \%$.

Results of sphingomyelin determination based on phosphorus content in TLC spots removed from the plate are presented in Table 4. 


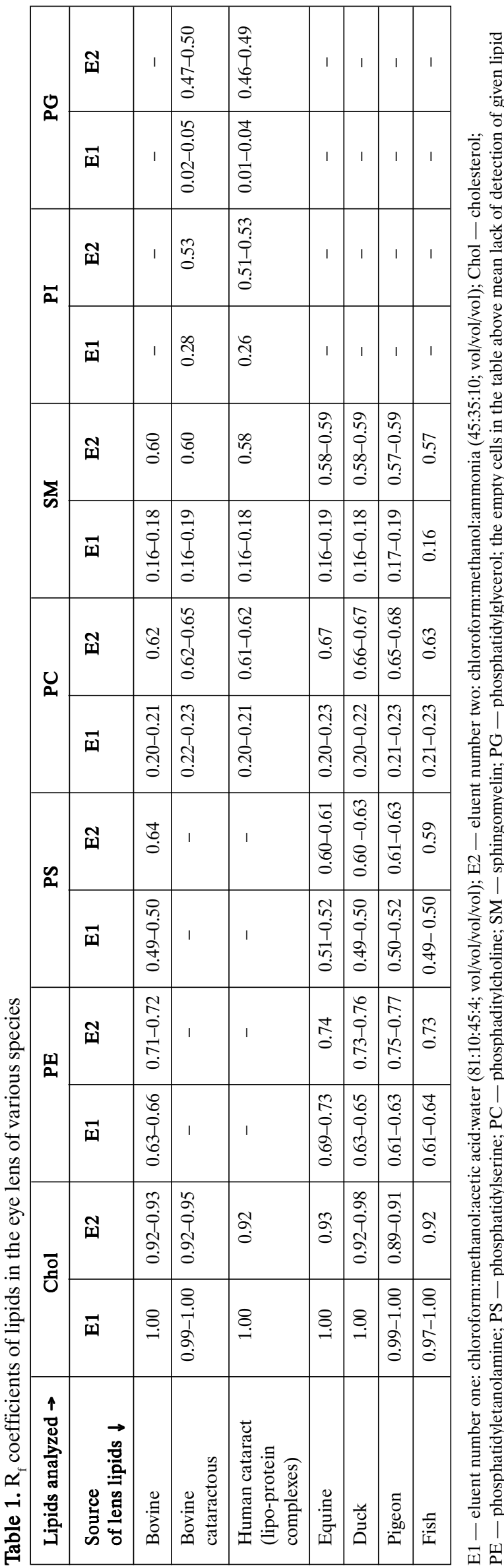

Table 2. Cholesterol content in lens lipids of various species - based on OPA (o-phtaldialdehyde) reaction, in percent of total lipid mass. For each measurement, the lipids were obtained from a separate lens

\begin{tabular}{|l|c|c|c|c|c|c|}
\hline $\begin{array}{l}\text { Sample of lens } \\
\text { lipids from: }\end{array}$ & \multicolumn{7}{|c|}{ Cholesterol content (\%) } \\
\hline Cow & 23.66 & 17.97 & 18.57 & 22.78 & 29.55 & - \\
\hline $\begin{array}{l}\text { Cow with } \\
\text { cataract }\end{array}$ & 16.73 & 17.54 & 29.55 & - & - & - \\
\hline Horse & 29.55 & 22.78 & 21.99 & 20.93 & 21.03 & 29.96 \\
\hline $\begin{array}{l}\text { Human cataract } \\
\text { lipo-protein } \\
\text { complexes }\end{array}$ & 32.98 & 27.72 & 31.16 & 35.57 & 17.76 & 14.01 \\
\hline Duck & 13.45 & 5.57 & 7.43 & 7.88 & - & - \\
\hline Fish & 5.57 & 4.37 & 3.58 & 2.89 & - & - \\
\hline
\end{tabular}

Table 3. Cholesterol content in lens lipids of various species - based on enzymatic assay, in percent of total lipid mass. For each measurement, the lipids were obtained from a separate lens

\begin{tabular}{|l|r|c|c|c|c|}
\hline $\begin{array}{l}\text { Sample of lens } \\
\text { lipids from: }\end{array}$ & \multicolumn{5}{|c|}{ Cholesterol content (\%) } \\
\hline Cow & 20.57 & 19.28 & 18.86 & 20.01 & 29.55 \\
\hline $\begin{array}{l}\text { Cow with } \\
\text { cataract }\end{array}$ & 14.65 & 18.3 & 24.12 & 16.69 & - \\
\hline Horse & 27.8 & 25.97 & 21.55 & 16.74 & 24.97 \\
\hline $\begin{array}{l}\text { Human cataract } \\
\text { lipo-protein } \\
\text { complexes }\end{array}$ & 35.22 & 32.2 & 14.1 & 12.39 & 28.03 \\
\hline Duck & 5.78 & 4.83 & 7.43 & - & - \\
\hline
\end{tabular}

Table 4. Sphingomyelin content in lens lipids of various species - based on phosphorus assay, in percent of total lipid mass. Each measurement was repeated twice on the same sample; the average is presented

\begin{tabular}{|l|c|}
\hline $\begin{array}{l}\text { Sphingomyelin fraction } \\
\text { derived from: }\end{array}$ & $\begin{array}{c}\text { Sphingomyelin } \\
\text { content (\% of total } \\
\text { lens lipid mass) }\end{array}$ \\
\hline Cow & 29.27 \\
\hline Cow with cataract & 25.85 \\
\hline Horse & 38.81 \\
\hline Human cataract lipo-protein complexes & 18.61 \\
\hline Fish & 17.98 \\
\hline
\end{tabular}

Results of sphingomyelin determination based on TLC densitometry are presented in Table 5.

The data of sphingomyelin content measurements presented in Tables 4 and 5 indicates small variation of the content of sphingomyelin among the samples 
Table 5. Sphingomyelin content in lens lipids of various species — based on TLC densitometry, in percent of total lipid mass. For each measurement, the lipids were obtained from a separate lens

\begin{tabular}{|l|c|c|c|c|c|c|}
\hline $\begin{array}{l}\text { Sphingomyelin } \\
\text { from eye lens of: }\end{array}$ & Exp. 1 & Exp. 2 & Exp. 3 & Exp. 4 & Average & $\begin{array}{l}\text { Standard } \\
\text { deviation }\end{array}$ \\
\hline Cow & 28.18 & 24.00 & 21.78 & 20.55 & 23.63 & 3.35 \\
\hline Cow with cataract & 22.29 & 21.01 & 20.56 & 19.71 & 20.89 & 1.08 \\
\hline Horse & 38.09 & 35.12 & 40.45 & 37.83 & 37.87 & 2.18 \\
\hline Human cataract lipo-protein complexes & 14.88 & 15.34 & 15.69 & 14.23 & 15.03 & 0.63 \\
\hline
\end{tabular}

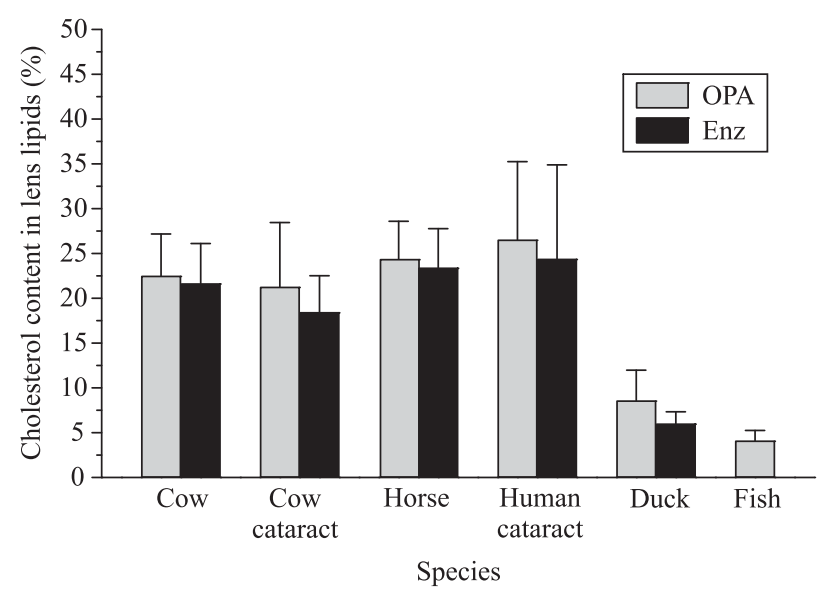

Figure 1. Cholesterol content in lens lipids of various species measured by two methods: OPA — spectrophotometric with phtalodialdehyde and ENZ - enzymatic with commercially available solution for cholesterol measurements in blood

derived in separate experiments within a given species. The highest sphingomyelin content was recorded for horse.

The averaged results of sphingomyelin assays are shown on Figure 2.

Two methods of sphingomyelin determination had shown similar results. The results of measurements performed with phosphorus test are higher than the results obtained by densitometry of sphingomyelin spots.

\section{Discussion}

The extraction procedure we used allowed the extraction of eye lens lipids and it turned out that for non-cataractous lenses of all investigated species, the detected phospholipids were the same, although in various proportions. The following lipids were detected: cholesterol, phosphatidylethanolamine, phosphatidylserine, phosphatidylcholine and sphingomyelin. The data presented in Table 1 for bovine lens lipids shows the qualitative change in phospholipid composition following cataract formation. The phosphatidylethanolamine and phosphatidylserine, present in all lens lipids derived from non-cataractous speci-

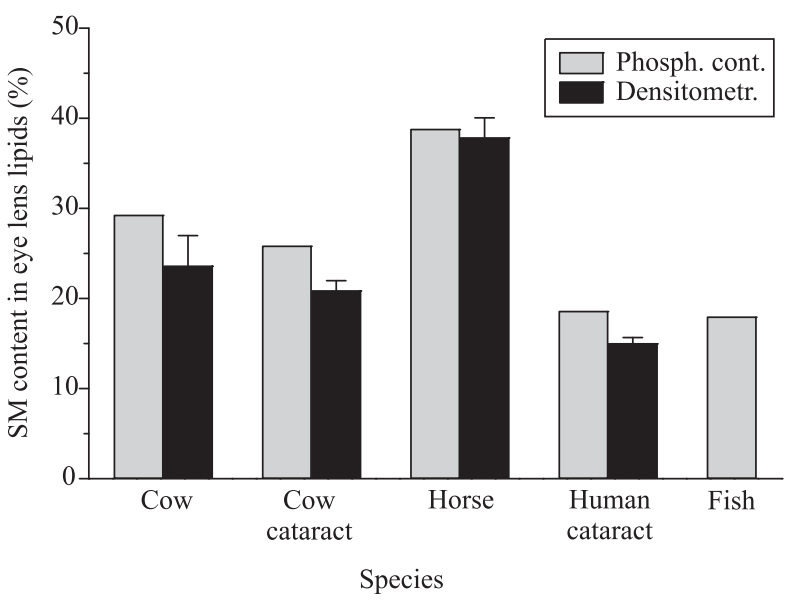

Figure 2. Sphingomyelin content in lens lipids of various species measured by two methods: by phosphorus content and by densitometry of SM spots in thin layer chromatography

TLC - thin layer chromatography; Chol - cholesterol; $\mathrm{PE}$ - phosphatidyletanolamine; PS - phosphatidylserine; PC - phosphaditylcholine; SM - sphingomyelin; PG - phosphatidylglycerol; OPA - phtalodialdehyde; eikonogen - eikonogen reagent $(25 \mathrm{mg}$ of sodium metabisulfite, $25 \mathrm{mg}$ of 1 amino 2 napthol 4 sulfonic acid and $1.49 \mathrm{~g}$ of sodium sulfite dissolved in $10 \mathrm{ml}$ of hot water, stored after filtration); $\mathrm{R}_{\mathrm{f}}$ - partition coefficient of substance detected by thin layer chromatography

mens, were not detected in cataract samples from either bovine or human specimens. On the other hand, samples derived from both bovine cataract and human cataract samples contained phosphatidylinositol and phosphatidylglycerol. These phospholipids were not present in lens lipids derived from clear, non-cataractous lenses. This observation indicates that during cataractogenesis some phospholipids undergo changes. It is well-known that phospholipids content change with age and cataract $[15,16]$. Some investigators have speculated that the changes in phospholipids content in lens lipids reflect the diminishing pool of glycerol-based lipids (phosphatidylcholines), which are usually unsaturated to a greater extent towards saturated sphingomyelins, especially dihydrosphingomyelin [15].

The methodology used in our work did not allow us to discern the degree of saturation of the lipids extracted, but it seems that the phospholipids bearing amino groups were replaced by other phospho- 
lipids, lacking easily dissociable protons. Such changes of phospholipids composition, if they occur in vivo, might affect the properties of lens fiber membranes crucial for lens homeostasis [16]. Moreover, the recent investigations of the Skulachev group indicate that charged molecules might play a crucial role in regulating the process of aging, including changes in the retina and eye lens $[17,18]$.

When the cholesterol content is analyzed, the smallest relative quantities were in the eye lens of a duck (about one third of the average relative cholesterol content of bovine and horse eye lens) and in the eye lens of fish (about one sixth of the relative cholesterol content in the eye lens content of lipids derived from the eye lens of mammals). Cholesterol might affect the interaction of lens membranes with alpha-crystallin, regarded as the key protein engaged in the protection of the vital lens fiber proteins [19].

The data for sphingomyelin relative content is incomplete, when compared to samples used for cholesterol determination, but even from that fragmentary data it can be seen that the relative sphingomyelin content is smallest in the fish eye lens. If sphingomyelin is regarded as the lipid protecting the peroxidation process in membranes [4, 20], this might reflect the relatively lesser danger of oxidative stress for aquatic vertebrates.

For mammals, the ratio of sphingomyelin:cholesterol molecules falls into the range $0.7-1.5$, and for the lipids of the eye lens of trout this ratio equals 0.23 , mainly due to much lower relative cholesterol content. Further investigations are necessary to determine whether such a low cholesterol: sphingomyelin ratio in eye lens lipids occurs in other aquatic species.

\section{References}

1. Vrensen G, Smeets M, Willekens B. The human eye lens is designed for longevity. Exp Eye Res. 1992;55(Suppl.):190.

2. Borchman D, Yappert MC, Afzal M. Lens lipids and maximum lifespan. Exp Eye Res. 2004;79:761-768.

3. Andrews SJ, Leonard-Martin T. Total lipid and membrane lipid analysis of normal animal and human lenses. Invest Ophth Vis Sci. 1981;21:39-45.
4. Yappert CM, Borchmann D. Sphingolipids in human lens membranes: an update on their composition and possible biological implications. Chem Phys Lipids. 2004;129: $1-20$.

5. Broekhuyse RM. Phospholipids in tissues of the eye: III. Composition and metabolism of phospholipids in human lens in relation to age and cataract formation. BBA — Lipid Lipid Met. 1969;187:354-365.

6. Definition of cataract - provided by ThinkQuest on-line library: Accessed 30 January 2010.

7. Bloemendal H, de Jong W, Jaenicke R, Lubsen NH, Slingsby $\mathrm{C}$, Tardieu A. Ageing and vision: structure, stability and function of lens crystallins. Prog Biophys Mol Bio. 2004;86: 407-485.

8. Hockwin O, Kojima M, Czubayko F, von Bergmann K. Inhibition of cholesterol synthesis and cataract. [Article in German] Fortschr Ophthal. 1991;88:393-395.

9. Cenedella RJ. Cholesterol and Cataracts. Surv Ophtalmol. 1996;40:320-337.

10. Folch J, Lees M, Sloane-Stanley GH. A simple method for isolation and purification of total lipids in animal tissues. J Biol Chem. 1957;226:497-509.

11. Colorimetric determination of free sterols. Website of CyberLipid Center: http://cyberlipid.org/cyberlip.home001.htm. Accessed 8 November, 2008.

12. Allain CC, Poon LS, Chan CSG, Ricmond W, Fu PC. Enzymatic determination of total serum cholesterol. Clin Chem. 1974;20:470-475.

13. Roeschlau P, Bernt E, Gruber WA. Enzymatische Bestimmung des Gesamt Cholesteiens im Serum. Z Klin Chem Klin Biochem. 1974;12:403-408.

14. Fiske $\mathrm{CH}$, Subbarow Y. Determination of Phosphorus. J Biol Chem. 1925;66:375-400.

15. Huang L, Grami V, Marrero Y et al. Human Lens Phospholipid Changes with Age and Cataract. Invest Ophth Vis Sci. 2005;46(5):1682-1689.

16 Zelenka PS. Lens lipids. Curr Eye Res. 1984;3:1337-1359.

17. Skulachev VP. A Biochemical Approach to the Problem of Aging: "Megaproject" on Membrane_Penetrating Ions. The First Results and Prospects. BIOCHEMISTRY (Moscow). 2007;72:1700-1714.

18. Skulachev VP, Anisimov VN, Antonenko YN et al. An attempt to prevent senescence: a mitochondrial approach. $B B A$ Bioenergetics. 2009;1787:437-461.

19. Tang D, Borchman D, Yappert MC, Cenedella RJ. Influence of cholesterol on the interaction of alpha-crystallin with phospholipids. Exp Eye Res. 1998;66:559-567.

20. Oborina EM, Yappert MC. Effect of sphingomyelin versus dipalmitoylphosphatidylcholine on the extent of lipid oxidation. Chem Phys Lipids. 2003;123:223-232.

Submitted: 8 February, 2010

Accepted after reviews: 1 March, 2011 